\title{
Condutas no paciente com insuficiência cardíaca internado em enfermarias de medicina interna
}

\section{Management of the inpatient with heart failure in an internal medicine ward}

Luis F. Joaquim ${ }^{1,2}$, Gustavo J. Volpe ${ }^{1,3}$, Alexandre B. de Figueiredo ${ }^{1,4}$, Julio C. Moriguti ${ }^{5}$

\begin{abstract}
RESUMO
A crescente prevalência da Insuficiência Cardíaca na população, e a constante má-aderência aos tratamentos propostos em nível ambulatorial, fazem com que o quadro de descompensação desta doença esteja entre as recordistas em número de internações, causando grande impacto na saúde pública do Brasil. O propósito deste estudo é o de estabelecer um protocolo de condutas a ser aplicado em pacientes com Insuficiência Cardíaca internados em enfermaria de Clínica Médica Geral, como o Hospital Estadual de Ribeirão Preto (SP, Brasil), com o objetivo de garantir a recuperação de um quadro de descompensação recente, assim como reorientar o paciente quanto à importância da aderência ao tratamento instituído. Para tanto, serão propostas a utilização e otimização de ferramentas farmacológicas, além de reintrodução de medidas não-farmacológicas, de modo que se reduza o tempo de internação, o número de recorrências, e favoreça o melhor seguimento ambulatorial destes pacientes.
\end{abstract}

Palavras-chave: Insuficiência Cardíaca. Quartos de Pacientes. Tratamento Clínico.

\section{Introdução}

Insuficiência cardíaca (IC) é definida como a incapacidade da bomba cardíaca em satisfazer a demanda metabólica dos tecidos quando em condições de volemia e/ou retorno venoso adequado, ou fazê-lo à custa de elevadas pressões de enchimento de suas câmaras ${ }^{1,2,3}$. Em geral, as manifestações clínicas poderão estar associadas tanto aos sintomas congestivos (congestão pulmonar e edema de membros inferiores), quanto aos sintomas de baixo débito cardíaco (fadiga muscular, disfunção renal), embora os pacientes comumente procurem o serviço médico com a queixa de dispneia secundária à perda progressiva da tole-
1 Médico Assistente da Área de Clínica Médica do Hospital Estadual de Ribeirão Preto.

2 Mestre em Fisiologia Cardiovascular pela Área de Fisiologia da FMRP-USP.

3 Pós-graduando da Área de Clínica Médica da FMRP-USP.

4 Mestre em Clínica Médica pela Área de Clínica Médica da FMRPUSP.

5 Diretor de Atividades Clínicas do Hospital Estadual de Ribeirão Preto e Professor Livre-Docente da Divisão de Clínica Médica Geral e Geriatria da FMRP-USP.
Correspondência: Luis Fernando Joaquim Hospital Estadual de Ribeirão Preto Avenida Independência, 4750 14026-160 - Ribeirão Preto/SP Telefone: 16-3602-7100 e-mail: ljoaquim1973@bol.com.br 
rância ao esforço físico ${ }^{1,2,3}$. Existem, ainda, circunstâncias em que a volemia e o débito cardíaco estão adequados, mas o indivíduo apresenta queixas sugestivas de IC devido à inadequação da função cardíaca às demandas metabólicas teciduais, como acontece em casos de hipertireoidismo, anemia, fístulas arteriovenosas, entre outros, correspondendo à IC de alto débito ${ }^{1,3}$.

A IC pode ser considerada como sistólica, quando secundária à menor fração de ejeção ventricular devido a um déficit de contração miocárdica, ou como de fração de ejeção preservada (antes conhecida como "diastólica"), secundária a certo grau de disfunção no relaxamento ventricular ${ }^{1,3,4}$. A IC sistólica corresponde a cerca de $60 \%$ dos casos, e pode estar associada à miocardiopatia de diversas etiologias (isquêmica, alcoólica, chagásica, entre outras) ou à cardiomiopatia dilatada ${ }^{2}$. Enquanto isso, a IC com fração de ejeção preservada se associa a uma série de fatores epidemiológicos, como idade maior que 65 anos, sexo feminino, hipertensão arterial, hipertrofia concêntrica do ventrículo esquerdo (VE), obesidade e, em menor proporção, ao diabetes e à doença isquêmica do coração ${ }^{5}$. É digno de nota que pacientes com o diagnóstico prévio de IC sistólica podem desenvolver, em certo momento da evolução natural de sua doença, disfunção diastólica significativa do VE, o que leva à piora sintomática e da qualidade de vida, além de reduzir a expectativa de vida ${ }^{3}$.

Em nível ambulatorial, os pacientes têm sido classificados quanto ao estadiamento da IC e o grau de limitação funcional imposta pela doença nas atividades do cotidiano ${ }^{1,4}$. A classificação em estágios da doença (A a D), conforme estabelecido pela American College of Cardiology (ACC) e American Heart Association (AHA) em 2005 (Tabela 1), objetiva acompanhar o desenvolvimento e a progressão da IC desde pacientes assintomáticos, mas com significativos fatores de risco para IC e/ou alterações estruturais cardíacas, até aqueles com quadros mais graves da doença, visando a escolha da melhor estratégia preventiva, terapêutica e de seguimento ambulatorial ${ }^{1,2,3,4}$. Por outro lado, a alocação dos pacientes em classes funcionais, proposta pela New York Heart Association (NYHA) (Tabela 2), visa estabelecer parâmetros clínicos para fins de prognóstico e seguimento clínico, permitindo-se avaliar a aderência ao tratamento e a resposta clínica à terapêutica farmacológica ${ }^{2}$. De acordo com este sistema de classificação, o paciente poderá mudar de classe funcional conforme houver regressão ou progressão de sua doença, como resposta à sua aderência ao tratamento proposto, embora este tipo de avaliação esteja sempre sujeita à interpretação subjetiva do profissional que o acompanha ${ }^{2}$.

A crescente prevalência da IC acompanha, atualmente, o envelhecimento da população brasileira. Considerada uma das principais causas de internação hospitalar de rotina ou emergência no momento, a IC se tornou um distúrbio de significativa morbimortalidade aos pacientes, com grande impacto nos custos para a saúde pública ${ }^{1,3}$. O cuidadoso manejo clínico ambulatorial permite adequado controle a longo-prazo dos sintomas da doença, mantendo-se o paciente funcionalmente ativo e com bom nível de qualidade de vida. Excluindo-se a má aderência ao tratamento, responsável pela grande maioria dos quadros

\section{Tabela 1}

Classificação da IC em estágios (A a D), conforme proposto pela American College of Cardiology (ACC) e American Heart Association (AHA) em 2005 (citado na referência 2)

\begin{tabular}{|c|c|c|}
\hline Estágio & Sintomas de IC & Descrição \\
\hline $\mathbf{A}$ & Ausentes & Pacientes com fatores de risco para o desenvolvimento de $\mathrm{IC}^{1}$ \\
\hline B & Ausentes & Pacientes com alterações estruturais cardíacas que predispõem à IC ${ }^{2}$ \\
\hline $\mathbf{C}$ & Presentes & $\begin{array}{l}\text { Pacientes com sintomas atuais ou pregressos de IC associados a alterações } \\
\text { estruturais cardíacas }\end{array}$ \\
\hline D & Presentes & Pacientes com sintomas refratários ao tratamento clínico otimizado ${ }^{3}$ \\
\hline
\end{tabular}




\section{Tabela 2}

Classificação do grau de limitação funcional dos pacientes com IC de acordo com a New York Heart Association (NYHA).

\begin{tabular}{cl}
\hline Classe Funcional & Descrição \\
\hline I & Sintomas ocorrem em níveis de esforço físico que limitariam até indivíduos normais \\
II & Sintomas ocorrem em níveis de esforço físico encontrados nas atividades do cotidiano \\
III & Sintomas ocorrem aos pequenos esforços \\
IV & Sintomas ocorrem aos mínimos esforços ou mesmo durante o repouso \\
\hline
\end{tabular}

de descompensação da IC, outras causas da piora clínica devem sempre ser investigadas. Após o período crítico de descompensação, não tendo havido causa orgânica para a mesma, pacientes com IC podem permanecer internados em hospitais de média complexidade para receber orientações e ter suas medicações reajustadas, a fim de que retornem à classe funcional ao qual pertenciam.

O presente estudo tem como objetivo atualizar os protocolos do Hospital Estadual de Ribeirão Preto (HERP), um hospital de nível secundário, a fim de que sejam revistas as orientações do paciente sobre as medidas não-farmacológicas, além de otimizadas as medicações para o controle da IC, com o propósito do mesmo retornar ao ambulatório especializado com controle mais adequado de sua doença. Para tanto, recomendações recentemente publicadas em consenso nacional ${ }^{1}$ e internacionais ${ }^{2,4}$ serão discutidas e adaptadas ao protocolo proposto, não sendo abordados os dispositivos utilizados para o controle de arritmias ou ressincronização cardíaca, visto não serem de realização em enfermarias de média complexidade. Embora hoje se reconheça a importância da IC com fração de ejeção normal, este capítulo terá como foco apenas o manejo clínico da IC sistólica. Além disso, detalhes acerca do quadro clínico, investigação complementar e diagnóstico diferencial da IC são amplamente abordados em livros texto ${ }^{3,6}$, de modo que somente aspectos relativos ao manejo clínico serão discutidos no presente texto.

\section{Manejo clínico e multidisciplinar do paciente com IC}

Os principais objetivos do tratamento da IC são o alívio dos sintomas, a melhora na qualidade de vida e na sobrevida do paciente, e a prevenção da progres- são da doença ${ }^{7}$. Em todos os estágios da IC, as medidas não-farmacológicas correspondem à base do tratamento, sem as quais o controle dos fatores de risco, e o uso de medicações para o controle dos sintomas, tendem a se tornar ineficazes e o paciente a progredir em sua doença. Neste contexto, a abordagem multidisciplinar, com a participação ativa de profissionais das áreas de Nutrição, Farmácia Hospitalar, Fisioterapia, Psicologia, Assistência Social e Terapia Ocupacional, deve ser considerada como essencial para se ter sucesso no manejo clínico de todo paciente com IC. Com isso, orientações acerca da dieta mais apropriada, uso correto das medicações, exercícios físicos e respiratórios regulares, e atividades de lazer, além de adequada abordagem de distúrbios psicossociais, incluindo o envolvimento de todos os seus familiares mais próximos, devem constituir o cerne da aderência do paciente ao tratamento crônico de sua doença. Sem isso, até o uso de medicamentos de última geração e de alta tecnologia pode se tornar obsoleto e ineficaz para o paciente.

\subsection{Medidas não-farmacológicas}

Dentre as medidas não-farmacológicas estabelecidas, a dieta com restrição de sal é de vital importância para a diminuição da retenção hídrica, a qual se constitui em uma das principais características do paciente com IC. A intensidade da restrição de sódio poderá depender do estágio da doença, mas geralmente varia de 1 a 2 g de sal por refeição. Pacientes hipertensos já se beneficiarão da dieta hipossódica oferecida em condições de IC, e aqueles com insuficiência renal crônica poderão requerer restrição ainda maior de sal.

Quanto à restrição hídrica, recomenda-se a ingestão de líquidos em, no máximo, 1000 a $1500 \mathrm{~mL} /$ dia para aqueles pacientes com ganho significativo de 
peso e que estão hiponatrêmicos (sódio sérico abaixo de $130 \mathrm{mEq} / \mathrm{L}$ ) e/ou refratários ao uso de diuréticos ${ }^{1,8}$. Além disso, consensos médicos têm orientado a medida diária do peso corporal do paciente, em nível domiciliar, a fim de se permitir o uso de doses menores e mais seguras dos diuréticos, além de reduzir a frequiência de exacerbações dos sintomas congestivos e o número de internações no setor de emergência dos hospitais ${ }^{1,2}$.

A terapia dietética poderá também ser adequada aos diversos fatores de risco associados (p. ex. hipogordurosa, com baixo teor de açúcar de absorção rápida), mas especial atenção deverá ser dada ao risco de desnutrição em si, o qual existe principalmente nos pacientes com IC em fase terminal ${ }^{1}$.

Hábitos considerados como de risco para o sistema cardiovascular (tabagismo, etilismo, drogadição, abuso de medicamentos) deverão ser abordados durante a internação, tendo-se, como alternativa, a possibilidade de encaminhamento do paciente para serviços especializados no tratamento a longo-prazo de cada distúrbio. Ainda assim, suporte psicológico adequado poderá ser oferecido ao paciente e aos seus familiares ainda durante a internação corrente.

Estudos apontam para significativa piora na qualidade de vida e no prognóstico dos pacientes com IC que mantém certo grau de sedentarismo, embora apresentem boa aderência à dieta e ao tratamento medicamentoso otimizado ${ }^{2,8}$. Desta forma, atividade física programada, sob a supervisão médica ou de um profissional fisioterapeuta, deveria ser oferecida com o objetivo de se manter o condicionamento físico (exercícios aeróbicos) e adequada massa muscular (exercícios isométricos $)^{9,10}$.

O consenso publicado pela ACC/AHA em 2009 reforça a orientação de que todos os pacientes com sinais e/ou sintomas de IC deveriam receber imunizações anti-influenza e anti-pneumococo ${ }^{2}$. Sendo assim, todo paciente com o diagnóstico de IC admitido na enfermaria do HERP deveria ter sua carteira de vacinação checada, e receber as respectivas atualizações das vacinas em atraso.

Finalmente, considerando-se que significativa parcela dos pacientes com IC admitidos no HERP apresentam baixas condições socioeconômicas e culturais, um serviço de Assistência Social atuante torna-se imprescindível, de modo que os mesmos tenham o devido acesso a medicações de alto custo e aos serviços especializados disponíveis no Sistema Único de Saúde, entre outros benefícios.

\subsection{Controle de fatores de risco e doenças associadas à IC}

Pacientes com IC no estágio A e B (Tabela 1) são considerados de grande risco para o desenvolvimento de doença sintomática, o que torna essencial a pronta identificação de tais situações nos indivíduos admitidos na enfermaria do HERP, e não apenas naqueles com suspeita ou diagnóstico já confirmado de IC. Nestes casos, as orientações ao paciente e o controle dos fatores de risco cardiovasculares deverão fazer parte da abordagem de rotina durante as internações.

Desta forma, pacientes hipertensos deveriam ser mantidos com a pressão arterial nos menores níveis possíveis, especialmente aqueles que também são diabéticos ${ }^{2,8}$, e como não é incomum a associação de outras comorbidades (coronariopatia, diabetes, insuficiência renal crônica), o esquema terapêutico para o controle pressórico deveria respeitar as principais indicações e contraindicações para as respectivas doenças ${ }^{2}$.

O diabetes mellitus também aumenta o risco de desenvolvimento de IC e, inclusive, determina pior prognóstico nos pacientes que já têm diagnóstico estabelecido. Por esse motivo, o controle rigoroso dos níveis glicêmicos é o objetivo a ser alcançado em todos os casos admitidos na enfermaria do HERP, devendo-se considerar o início de insulinoterapia naqueles indivíduos que se encontram em dose máxima dos hipoglicemiantes orais disponíveis na rede pública de saúde, ou que tenham contraindicações absolutas para os mesmos (p.ex., pacientes renais crônicos) ${ }^{2}$.

Outros fatores de risco cardiovascular, como obesidade, uso do tabaco, etilismo crônico, uso de drogas psicoativas (p. ex. cocaína, anfetaminas), e distúrbios como síndrome metabólica e coronariopatia, merecem também abordagem destacada durante eventual internação na enfermaria do HERP. Nestes casos, orientações multidisciplinares e medidas farmacológicas preventivas (AAS, hipolipemiantes, adesivos de nicotina), deverão ser oferecidas desde a admissão do paciente, e uma programação de Alta Hospitalar deverá ser instituída para que o paciente possa ter continuidade do tratamento em sua cidade de origem.

\subsection{Arsenal farmacológico e suas indicações}

À medida que o paciente adquire lesão estrutural cardíaca, a escolha da terapêutica medicamentosa 
dependerá não apenas do estadiamento em que ele se encontra (estágios B a D - Tabela 1), mas também da classe funcional na escala do NYHA (Tabela 2), com ajustes nas doses conforme piora nesta última $^{1,2,4,7,11,12}$. Assim, pacientes assintomáticos serão beneficiados com o uso de medicamentos que bloqueiem o eixo neuro-hormonal, com efeito inibitório sobre o remodelamento adverso dos ventrículos ${ }^{7}$. Por outro lado, pacientes que se tornam sintomáticos podem apresentar quadro congestivo sistêmico e/ou pulmonar, e se beneficiarão também com diuréticos e inotrópicos positivos ${ }^{7}$. A seguir, serão revisados os principais conceitos e aplicações do arsenal farmacológico utilizado no manejo clínico da IC. Os fármacos de cada classe a seguir, suas respectivas doses iniciais e máximas, entre outros detalhes, estão descritos na Tabela 3.

\subsubsection{Drogas que interferem no remodelamento miocárdico}

\section{- Inibidores da Enzima Conversora de Angioten- sina}

Os medicamentos que inibem a enzima conversora de angiotensina I em angiotensina II (IECA) demonstram vários benefícios no tratamento da IC, agindo no alívio dos sintomas, na melhora da classe funcional e na sensação de bem-estar dos pacientes. Além disso, o IECA pode reduzir o risco de hospitalização e morte, o que implica em melhora significativa no prognóstico, independentemente da presença de doença coronariana. Deste modo, o IECA deverá ser prescrito para todos os casos de IC devido a uma disfunção sistólica do VE, a menos que haja alguma contraindicação ou intolerância por parte do paciente pr,2,4,12 $_{\text {. }}$

\section{Tabela 3}

Fármacos mais usados no Brasil para manejo da IC crônica, e respectivas posologia, doses iniciais e máximas (ou alvo $)^{1}$

\begin{tabular}{|c|c|c|c|}
\hline Droga & Dose Inicial (mg) & Dose Alvo ou Máxima (mg) & Frequência (vezes/dia) \\
\hline \multicolumn{4}{|l|}{ Inibidores da ECA/BRAs } \\
\hline Captopril & 6,25 & 50 & 3 \\
\hline Enalapril & 2,5 & 20 & 2 \\
\hline Losartan & 25 & $50-100$ & 1 \\
\hline Valsartan & 40 & 320 & 2 \\
\hline \multicolumn{4}{|l|}{ Beta-bloqueadores } \\
\hline Bisoprolol & 1,25 & 10 & 1 \\
\hline Carvedilol & 3,125 & 50 se $\mathrm{P}<85 \mathrm{Kg} ; 100$ se $\mathrm{P}>85 \mathrm{Kg}$ & 2 \\
\hline Nebivolol & 1,25 & 10 & 1 \\
\hline Succinato metoprolol & 12,5 & 200 & 1 \\
\hline \multicolumn{4}{|l|}{ Inibidores Aldosterona } \\
\hline Amilorida & 2,5 & $20 *$ & 1 \\
\hline Espironolactona & 25 & $50 *$ & 1 \\
\hline \multicolumn{4}{|l|}{ Diuréticos de Alça } \\
\hline Bumetanida & $0,5-2$ & $10 *$ & Variável \\
\hline Furosemida & 20 & $240 *$ & Variável \\
\hline \multicolumn{4}{|l|}{ Diuréticos Tiazídicos } \\
\hline Hidroclorotiazida & 25 & $100 *$ & 1 \\
\hline Metolazona & 2,5 & $10 *$ & 1 \\
\hline \multicolumn{4}{|l|}{ Demais medicações } \\
\hline Digoxina & $0,125-0,25$ & $\#$ & $\#$ \\
\hline Amlodipina & 2,5 & 10 & 1 \\
\hline
\end{tabular}

* doses máximas

\# dose máxima e frequiência variáveis, podendo-se utilizar doses em dias alternados

$\mathrm{P}=$ peso $(\mathrm{kg})$

$\mathrm{ECA}=$ enzima de conversão da angiotensina; $\mathrm{BRA}=$ bloqueadores dos receptores de angiotensina 
Na prática clínica, há poucas diferenças entre os vários IECA no que diz respeito aos seus efeitos sobre os sintomas ou a sobrevida do paciente. Apesar de seu efeito vasodilatador ser importante na redução da pós-carga no quadro de IC, considera-se que a principal ação do IECA seja a de bloquear os efeitos deletérios do sistema renina-angiotensina-aldosterona no remodelamento do tecido cardíaco ${ }^{1,2,7}$. Por este motivo, sempre que possível, o IECA deveria ser prescrito em todos os pacientes com IC no estágio C e D, preferencialmente associado à droga bloqueadora betaadrenérgica, mesmo que a dose máxima desta última ainda não tenha sido atingida ${ }^{2}$.

A terapêutica com o IECA deverá ter início com doses baixas, seguindo-se incremento na dose a cada 3-5 dias conforme a tolerância do paciente ${ }^{1,2,7}$. Cerca de 1-2 semanas após a introdução do IECA, o paciente deverá ser avaliado quanto a possíveis efeitos adversos, como hipotensão (inclusive de caráter postural), piora da função renal e hipercalemia; reações adversas muito graves, como angioedema e falência renal, são raras e exigem a sua suspensão imediata $^{2}$. A meta do tratamento com o IECA deverá ser o de atingir as doses terapêuticas preconizadas por estudos multicêntricos ("trials"), desde que bem toleradas pelo paciente e sem efeitos adversos significativos. Se houver hipotensão arterial, piora da função renal ou hipercalemia, desde que sem risco de morte para o paciente, a dose diária do IECA deverá ser reajustada para baixo, mas sem suspendê-lo, visto que a sua retirada abrupta poderá deteriorar o quadro clínico do paciente ${ }^{2,7}$.

Pacientes que ainda estejam em fase de compensação de quadro congestivo poderão perder transitoriamente os efeitos benéficos do IECA devido à acentuada retenção hídrica. Neste caso, torna-se importante a otimização da terapia com diuréticos antes da introdução do IECA, embora possa ser necessária a redução do diurético prescrito no momento exato do início do IECA, para que se previna uma eventual hipotensão sintomática ${ }^{7}$. Por outro lado, em pacientes com instabilidade hemodinâmica, a manutenção do IECA poderá prejudicar a resposta natriurética dos diuréticos e diminuir a resposta pressora de vasoconstritores arteriolares, de modo que a medicação deverá ser suspensa, e retornada tão logo o paciente tenha o quadro clínico completamente estabilizado ${ }^{2}$.

Quanto aos efeitos adversos do IECA, hipotensão e tontura são os mais comuns, principalmente nos primeiros dias de início da terapêutica. Desde que o episódio de hipotensão não ocorra com sintomas posturais acentuados, piora da função renal ou síncope, o IECA poderá ser mantido nas mesmas doses, e outras medicações anti-hipertensivas (incluindo diuréticos) deverão ter suas doses reajustadas ou seus horários de administração alterados ${ }^{2}$.

A introdução do IECA poderá levar a uma piora significativa da função renal, medida pelos níveis de creatinina e uréia séricas. Neste caso, desde que o paciente não tenha estenose bilateral das artérias renais, o consenso norte-americano mais recente ${ }^{2}$ estabelece que as doses diuréticas devam ser reduzidas, e que um grau de azotemia leve a moderado pode ser tolerado, mesmo que não seja possível o ajuste no diurético, objetivando-se a manutenção do IECA. Por outro lado, se hipercalemia progressiva se desenvolver, com riscos para distúrbio de condução cardíaco, o IECA deverá ser suspenso e substituído por outros vasodilatadores ${ }^{2}$.

Cerca de $10 \%$ dos pacientes que iniciam o uso do IECA poderão se queixar, dentro dos primeiros meses da terapia, de tosse seca. Este quadro geralmente desaparece em até 2 semanas após a suspensão do IECA, mas preconiza-se esta conduta apenas se o paciente estiver sofrendo agravos na sua qualidade de vida, visto o enorme benefício da medicação para o manejo da $\mathrm{IC}^{2}$.

As contraindicações ao uso do IECA são: hipotensão arterial com pressão sistólica abaixo de 80 $\mathrm{mmHg}$, níveis acentuados de creatinina séricos $(>3$ $\mathrm{mg} / \mathrm{dL}$ ), hipercalemia acima de 5,5 mEq/L, angioedema prévio, e estenose bilateral de artérias renais ${ }^{1,2,4}$.

\section{- Antagonistas dos Receptores de Angiotensina II (ARA-II)}

Pacientes que não conseguem tolerar o IECA, devido à tosse associada a esta medicação ou a episódio prévio de angioedema, podem se beneficiar com a introdução de droga bloqueadora do receptor AT1 da angiotensina II (losartan, valsartan, candesartan, irbesartan, olmesartan). Por não inibir a enzima conversora de angiotensina, esta classe de medicamentos não promove o acúmulo de cininas, as quais são consideradas parte essencial na etiologia da tosse ou do angioedema nos pacientes em uso de IECA ${ }^{2,7}$.

Os consensos apontam que o ARA-II apresenta praticamente os mesmos benefícios que o IECA no controle do remodelamento cardíaco, o que significa redução nos índices de hospitalização e mortalidade ${ }^{2,7}$. Apesar disso, considera-se hoje que parte dos efeitos 
do IECA no quadro de IC crônica seja devido à hiperprodução de cininas, e não apenas ao bloqueio da produção de angiotensina II, de modo que cada vez mais se preconiza o uso concomitante do ARA-II com o IECA no manejo de pacientes com IC ${ }^{2,7}$. Além disso, o efeito adicional do ARA-II pode ser explicado pela presença de um escape parcial da inibição da enzima que acontece em pacientes em uso crônico do IECA, os quais apresentam níveis significativos de angiotensina II circulante apesar do uso regular do IECA ${ }^{2,7}$.

O ARA-II é habitualmente bem tolerado pelo paciente, e as doses iniciais devem ser as menores possíveis, com aumento gradual até que a dose plena da medicação seja atingida. As complicações associadas ao ARA-II são semelhantes àquelas observadas com o IECA (hipotensão arterial sintomática, alteração na função renal e hipercalemia), de modo que os mesmos cuidados descritos acima para o IECA devem ser aplicados quando do início do ARA-II ${ }^{2,7}$.

\section{- Beta-bloqueadores}

As drogas com ação bloqueadora dos receptores beta-adrenérgicos constituem um dos pilares no manejo do paciente com IC, principalmente aqueles com FE reduzida. Os beta-bloqueadores, como são denominados, diminuem os efeitos deletérios do sistema adrenérgico sobre o tecido e o funcionamento cardíacos, prevenindo e/ou reduzindo o remodelamento cardíaco, as arritmias, e a influência de outros eixos neuro-hormonais sobre o coração ${ }^{1,2,7,8}$. Quando prescritos juntamente com o IECA ou BRA, os beta-bloqueadores melhoram a qualidade de vida e a sobrevida dos pacientes, sejam eles sintomáticos ou não, com ou sem doença arterial coronariana ou diabetes mellitus, considerando-se ambos os sexos e as diversas etnias $^{2,7}$.

De toda a gama de beta-bloqueadores disponíveis no mercado, apenas 3 deles são atualmente aprovados para o uso em pacientes com IC: carvedilol $^{13}$, succinato de metoprolol e bisoprolol ${ }^{2}$. O succinato de metoprolol caracteristicamente proporciona uma liberação mais lenta e contínua do sal, quando comparado com o tartarato de metoprolol, o que poderia explicar o seu maior benefício no tratamento de manutenção da $\mathrm{IC}^{2}$; entretanto, no Brasil, apenas o tartarato de metoprolol é atualmente liberado para pacientes conveniados do Sistema Único de Saúde (SUS).

Assim como os IECAs, os beta-bloqueadores devem ser iniciados em baixas doses, com pequenos incrementos realizados a cada 7-14 dias, de modo a se atingir a dose efetiva registrada nos estudos multicêntricos ou, pelo menos, aquela que for tolerada pelo paciente $^{1,2,7}$. Este aumento gradual é importante para que não haja deterioração do quadro clínico do paciente na fase inicial do tratamento da IC, em que a súbita inibição do sistema adrenérgico poderia acarretar significativa instabilidade hemodinâmica ${ }^{7}$. Apesar disso, deve-se alertar o paciente de que os sintomas associados à IC podem piorar nos primeiros dias da introdução do beta-bloqueador (em até $15 \%$ dos casos) ${ }^{1,7}$, e que os resultados clínicos poderão demorar até 2-3 meses para acontecer, fatos que não devem em hipótese alguma levar a uma interrupção no tratamento com o beta-bloqueador ${ }^{2}$.

$\mathrm{O}$ consenso recentemente publicado pela ACC/ $\mathrm{AHA}^{2}$ preconiza que os médicos devem orientar seus pacientes a monitorizar um possível ganho de peso durante a fase de introdução do beta-bloqueador, que pode ocorrer devido ao aumento na retenção hídrica. Neste caso, os pacientes devem ser orientados a aumentar a dose dos diuréticos até a resolução do quadro congestivo, o que geralmente melhora a tolerância ao beta-bloqueador. Mesmo em casos de descompensação da IC, recomenda-se que não se suspenda abruptamente o beta-bloqueador, devendo-se otimizar a terapia diurética com doses endovenosas, a não ser que haja hipotensão e/ou sinais de hipoperfusão periférica, quando se recomenda a redução pela metade, ou mesmo a suspensão, do bloqueador beta-adrenérgico $^{2}$. Porém, se na fase inicial do tratamento surgir hipotensão arterial com sinais de hipoperfusão periférica, bloqueios de alto grau na condução cardíaca, ou sinais de broncoespasmo grave, o beta-bloqueador deverá ser prontamente suspenso ${ }^{2}$.

O beta-bloqueador deve ser prescrito para todos os pacientes com IC sistólica, podendo ser introduzido quando os mesmos estão em fase inicial do tratamento com IECA, desde que não estejam congestos ou hipotensos, ou ainda internados em Unidade de Terapia Intensiva recebendo drogas vasoati$\operatorname{vas}^{2,7}$. Conforme discutido acima, pacientes com quadro de retenção hídrica devem ser tratados primeiramente com diuréticos e, somente após compensação do quadro congestivo pulmonar e/ou sistêmico, iniciar terapia com o beta-bloqueador, mantendo-se ainda o uso do diurético ${ }^{2}$. Da mesma forma, pacientes com hipotensão sustentada, ou necessidade de suporte intensivo, devem receber terapêutica apropriada, e somente depois ser reavaliados para elegibilidade ao betabloqueador $^{2,7}$. 
Estudos multicêntricos têm demonstrado que a terapia beta-bloqueadora é bem tolerada pela maioria dos pacientes com IC, independentemente de comorbidades como diabetes mellitus, DPOC ou doença vascular periférica ${ }^{7}$. Assim, há uma tendência para se liberar o tratamento a pacientes com diagnóstico conhecido de DPOC, desde que os mesmos não tenham um quadro grave de hiperreatividade brônquica ${ }^{2}$. Apesar disso, contraindicações absolutas ao uso de betabloqueadores ainda existem, e consistem em bradicardia sintomática, bloqueios atrioventriculares de alto grau, asma ou DPOC grave g $^{1,2}$.

As doses utilizadas dos beta-bloqueadores no manejo da IC estão disponíveis na Tabela 3. É digno de nota que o nebivolol é um beta 1 -bloqueador adrenérgico bastante cardiosseletivo com efeito vasodilatador periférico mediado pela inibição da enzima NO sintetase endotelial (eNOS), diferentemente do carvedilol, que induz o mesmo efeito mas por meio da inibição $\alpha_{1}$-adrenérgica ${ }^{14,15}$. Por agir sobre a eNOS, o nebivolol apresenta propriedades antioxidantes, reverte disfunções endoteliais, e induz "downregulation" em processos inflamatórios, estresse oxidativo e proliferação de células musculares lisas, todas averiguadas em modelos experimentais, mas com potencial benefício clínico que poderá futuramente superar ao dos demais medicamentos desta classe ${ }^{14,15}$.

\section{- Antagonistas da Aldosterona}

Aldosterona, produzida principalmente nas glândulas supra-renais, mas também em células endoteliais no tecido cardíaco, após estimulação pela angiotensina-II, tem como principal mecanismo de ação o efeito mineralocorticóide de retenção de sódio e espoliação de potássio ${ }^{16}$. Tal efeito acontece nos túbulos contorcidos distais e coletores dos rins, no cólon, e nas glândulas sudoríparas e salivares, e funciona essencialmente como adaptação do organismo a situações de privação de $\mathrm{sal}^{7,16}$. Entretanto, em situações como a IC, o baixo débito cardíaco se constitui em estímulo para maior avidez ao sal, de modo que não apenas a produção de renina e angiotensina II, mas também a de aldosterona está aumentada ${ }^{16}$.

A elevação sérica de todos os hormônios do sistema renina-angiotensina-aldosterona é um dos principais mecanismos envolvidos no estado de hipervolemia, vasoconstricção periférica e fibrose intracardíaca em pacientes com $\mathrm{IC}^{2,16}$. Como discutido acima, o uso contínuo do IECA leva ao escape da produção de angiotensina II e, por conseguinte, à ativação da aldos- terona, de modo que um bloqueio da via final do sistema renina-angiotensina-aldosterona poderia resultar em efeito benéfico adicional na IC ${ }^{1}$.

O estudo multicêntrico RALES (Randomized Aldactone Evaluation Study), publicado em 1999, foi o primeiro a demonstrar que a inibição do efeito mineralocorticóide da aldosterona com espironolactona ( 25 a $50 \mathrm{mg} / \mathrm{dia}$ ) tinha impacto significativo não apenas na redução da mortalidade e do número de internações, mas também na melhora sintomática de pacientes com IC sistólica em classes funcionais III e IV, e que já faziam uso de IECA, diurético de alça e digoxina ${ }^{7}$. O efeito em longo prazo sobre a mortalidade poderia estar associado à redução na retenção de sódio e no remodelamento miocárdico, ambas as funções conhecidas da aldosterona ${ }^{2,7,16}$.

Embora estudos multicêntricos subseqüentes tenham mostrado os mesmos benefícios com outros inibidores da aldosterona ${ }^{7}$, a espironolactona se mantém como o principal agente desta classe de fármacos utilizada no Brasil. Ela está formalmente indicada para o tratamento de pacientes com IC em classes funcionais III e IV, em doses únicas diárias de 12,5 a $25 \mathrm{mg}$, desde que não haja histórico de insuficiência renal crônica (creatinina $>2,0 \mathrm{mg} \%$ ou depuração de creatinina $<30 \mathrm{~mL} / \mathrm{min}$ ) ou hipercalemia prévia, e que os valores de potássio sérico não estejam acima de $5,0 \mathrm{mEq} / \mathrm{L}$ no início do tratamento ${ }^{2}$.

O maior risco associado ao uso dos inibidores da aldosterona, diuréticos "poupadores de potássio", é o de hipercalemia potencialmente grave. Deste modo, após a introdução da espironolactona, o prescritor deverá se preocupar com os seguintes fatos: 1) há inibição da excreção de potássio pela própria medicação; 2) geralmente, há concomitante uso do IECA e BRA, medicações associadas ao risco aumentado de hipercalemia; e 3) inibidores da aldosterona, embora considerados diuréticos fracos, podem potencializar a ação dos demais diuréticos e levar ao estado de depleção volêmica, reduzindo ainda mais a excreção de potássio pela piora na função renal ${ }^{2}$. Assim, recomenda-se que se suspenda eventual reposição de potássio que esteja prescrita, e se avalie a dose diária de furosemida, antes da introdução da espironolactona na terapêutica do paciente com IC. Caso o paciente esteja em uso de IECA e de BRA juntos, recomenda-se a suspensão de um destes fármacos, em vista da potencialização do risco de hipercalemia ${ }^{2}$.

Após os cuidados acima, recomenda-se a monitorização dos níveis séricos de potássio após 3 e 7 
dias da introdução da espironolactona, seguidos por medidas mensais durante os 3 primeiros meses e, depois, a cada 3 meses de tratamento ${ }^{2}$. A introdução de IECA ou BRA, ou o aumento na dose destes medicamentos, implicará em novo ciclo de monitorização da calemia do paciente. A medicação deverá ter a sua dose reduzida pela metade, ou ainda poderá ser suspensa em definitivo, caso níveis deletérios de calemia sejam identificados durante este processo ${ }^{2}$.

É digno de nota que um dos efeitos adversos mais comumente associados ao uso da espironolactona é a ginecomastia, muitas vezes dolorosa e potencial causa da suspensão do tratamento. A substituição da espironolactona pela eplerenona (25 a $50 \mathrm{mg} / \mathrm{dia}$ ), inibidor da aldosterona de classe mais recente, poderá reduzir significativamente este efeito ${ }^{2}$.

\subsubsection{Diuréticos de Alça e Tiazídicos}

Diuréticos de alça, como furosemida, bumetanida e torsemida, são elementos essenciais na terapêutica de pacientes com IC e que apresentam sobrecarga de volume ou histórico de retenção hídrica. Eles atuam inibindo o co-transportador $\mathrm{Na}^{+}-\mathrm{K}^{+}-2 \mathrm{Cl}^{-}$da alça ascendente de Henle, levando a significativa espoliação eletrolítica e de água livre ${ }^{2,7}$. Preconiza-se o uso do diurético de alça todas as vezes que o paciente apresente congestão pulmonar e/ou sistêmica, podendo-se manter a medicação após a recuperação completa do quadro se o indivíduo tiver histórico de retenção hídrica, programando-se a otimização das doses de IECA e beta-bloqueador e a introdução, ou não, da espironolactona e do digitálico ${ }^{2,7}$. Caso contrário, o diurético deverá ser suspenso e reintroduzido apenas se recidiva do quadro congestivo, com o paciente fazendo o controle de seu peso diário de maneira rigorosa ${ }^{2}$.

Furosemida é o diurético de alça mais utilizado em nosso meio, tendo biodisponibilidade oral de 40 a $80 \%$, e meia-vida bastante curta (menos que 6 horas), o que eventualmente demanda doses frequentes em 24 horas para a sua maior eficácia ${ }^{7}$. Em situações de descompensação dos sintomas congestivos, doses parenterais de furosemida são preferencialmente prescritas, pois proporcionam recuperação mais rápida do paciente. Outras situações em que a via parenteral é preferida em relação à oral são os quadros de anasarca e choque circulatório, em que a absorção oral da furosemida se encontra comprometida por acentuado edema e redução da perfusão das alças intestinais, respectivamente, com as doses endovenosas sendo prescritas de forma intermitente ou em bomba de infusão contínua. ${ }^{2}$
Como os diuréticos de alça atuam inibindo o cotransportador $\mathrm{Na}^{+}-\mathrm{K}^{+}-2 \mathrm{Cl}^{-}$da alça ascendente de Henle, seu uso crônico pode levar a distúrbios eletrolíticos como hipocalemia, hiponatremia e hipomagnesemia ${ }^{7}$. Especial atenção deve ser dada à hipocalemia refratária às reposições orais ou parenterais de $\mathrm{KCl}$, em vista da possibilidade de hipomagnesemia ou alcalose metabólica concomitantes ${ }^{7}$. Alguns destes distúrbios predispõem o paciente a arritmias cardíacas, principalmente se ele estiver em uso de fármacos como amiodarona, lidocaína ou quinidina; por isso mesmo, os níveis séricos de potássio devem ser mantidos entre 4 e $5 \mathrm{mEq} / \mathrm{L}$ em pacientes com IC e que estejam em terapia diurética ${ }^{7}$.

Durante a terapia com diuréticos de alça, o paciente poderá apresentar hipotensão arterial e/ou piora da função renal. Neste caso, desde que o paciente se mantenha assintomático durante o episódio hipotensivo e o aumento nas escórias nitrogenadas permaneça em níveis aceitáveis, o uso dos diuréticos não deverá ser interrompido e nem a dose deverá ser reduzida, priorizando-se a resolução do estado congestivo do paciente ${ }^{2}$. Além disso, atenção especial deverá ser dada à otimização da terapêutica vasodilatadora e à recirculação do edema presente nos membros inferiores do paciente. A prioridade em se resolver o quadro congestivo do paciente se faz na tentativa de se melhorar a absorção dos fármacos prescritos por via oral, o que levaria a maior eficácia dos vasodilatadores e medicamentos que melhoram a sobrevida do paciente (IECA, beta-bloqueador e espironolactona) ${ }^{2}$

Eventualmente, o paciente com IC em uso crônico de diurético de alça pode se tornar resistente à ação desta classe de medicamentos. Esta resistência pode ser um fenômeno multifatorial e se caracterizar pela diminuição da resposta diurética e natriurética mesmo com doses moderadas de furosemida ${ }^{7}$. Neste caso, os potenciais fatores envolvidos são os seguintes: 1) diminuição da resposta intrínseca da natriurese após certa dose do diurético, mesmo por via parenteral (efeito "braking"); 2) contração do volume intravascular causada pelo uso excessivo do diurético, levando à redução da pressão de perfusão renal e da taxa de filtração glomerular; 3) piora progressiva da função renal por outras causas (comorbidades, uso de drogas como anti-inflamatórios não-esteroidais); 4) menor biodisponibilidade do diurético por via oral (em nível ambulatorial) em situações de congestão sistêmica; 5) má-aderência à terapêutica diurética; 6) otimização das drogas vasodilatadoras em pacientes com estenose de artéria renal suspeita ou confirmada, o 
que geralmente leva à piora na perfusão renal; 7) desenvolvimento da síndrome cardiorrenal, de etiologia ainda desconhecida ${ }^{7}$.

Ainda que o paciente com IC apresente indícios de resistência aos diuréticos de alça, uma estratégia que se tem mostrado eficaz em aumentar o volume urinário é a associação de altas doses de um diurético tiazídico ao esquema terapêutico. Desta forma, hidroclorotiazida pode ser adicionada em doses únicas diárias de 50 a $100 \mathrm{mg}$, devendo-se monitorizar cuidadosamente a resposta diurética e o peso em jejum, assim como a função renal e a natremia do paciente $^{7}$. Outra maneira de se superar a resistência ao uso dos diuréticos de alça é a administração da dose diária prescrita via bomba de infusão contínua, fazendo-se a injeção em bolus de 20 a $40 \mathrm{mg}$, seguida pela infusão contínua de 5 a $10 \mathrm{mg} /$ hora $^{7}$.

\subsubsection{Digitálicos}

Glicosídeos cardíacos são fármacos que atuam inibindo a bomba $\mathrm{Na}^{+}-\mathrm{K}^{+}$-ATPase presente nas membranas celulares de todas as células. Antigamente, postulava-se que seu único mecanismo de ação nas células cardíacas era o de aumentar os níveis intracelulares de cálcio, determinando efeito inotrópico positivo. Entretanto, estudos recentes identificaram que os digitálicos não apenas exercem efeito inotrópico cardíaco, mas também que a sua ação sobre a bomba $\mathrm{Na}^{+}-\mathrm{K}^{+}$-ATPase leva ao aumento do tônus vagal sobre o sistema de condução cardíaco, determinando menor atividade do nó sinusal e também importante efeito cronotrópico negativo ${ }^{2,7}$. Além disso, seu mecanismo de ação nos túbulos renais leva à menor reabsorção tubular de sódio e, por conseguinte, a redução significativa na secreção de renina pelos rins, o que seria benéfico para o paciente com IC que caracteristicamente tem retenção de sódio e estimulação dos eixos neuro-hormonais, incluindo o sistema reninaangiotensina-aldosterona ${ }^{2,7}$.

Dos digitálicos disponíveis, o mais utilizado no manejo a longo prazo de pacientes com IC é a digoxina. Estudos multicêntricos têm sugerido que a introdução da digoxina no esquema terapêutico da IC não altera a mortalidade, embora induza significativa melhora na qualidade de vida do paciente, com redução nos sintomas, aumento na tolerância às atividades físicas, e menor número de internações hospitalares ${ }^{2,7}$. Apesar disso, o uso regular da digoxina tem se mantido importante tema de discussões entre os especialistas da área, que buscam inseri-la no aparato farmacológico ambulatorial da IC.
Atualmente, a digoxina tem sido considerada medicação com uso reservado para pacientes com IC que apresentem falha terapêutica após otimização das drogas que melhoram a sobrevida, como IECA, betabloqueador e aldosterona, assim como naqueles que já estejam em uso de doses moderadas de diuréticos ${ }^{2}$. Por outro lado, se algum paciente estiver em uso apenas de digoxina, recomenda-se que não se interrompa a sua prescrição e se associe as medicações que efetivamente bloquearão os eixos neuro-hormonais, como IECA e beta-bloqueador, respeitando-se as respectivas contraindicações ${ }^{2}$.

A diretriz brasileira para o manejo de IC estabelece indicação formal da digoxina (recomendação classe I) em pacientes com IC sistólica e que apresentem fibrilação atrial com elevada resposta ventricular, desde que as demais medicações já estejam com suas doses otimizadas ${ }^{1}$. Por outro lado, a diretriz norteamericana considera que as medicações beta-bloqueadoras são as que melhor controlam a resposta ventricular de frequência cardíaca, e o uso concomitante de digoxina em pacientes "beta-bloqueados" poderia incorrer em risco elevado de arritmias cardíacas, de modo que, mesmo para tais circunstâncias, a recomendação atual foi rebaixada para classe IIa (vide textos das diretrizes citadas para compreensão das Classes de Recomendação atualmente em uso) ${ }^{2}$. Sendo assim, torna-se plausível a utilização dos digitálicos nos quadros de descompensação da IC em que os beta-bloqueadores estão formalmente contraindicados.

Definindo-se o paciente como elegível para o tratamento com digoxina, a medicação deveria ser prescrita em doses únicas diárias de 0,125 a 0,250 mg. A dose de ataque, antigamente considerada como necessária para "impregnação do paciente", não é mais preconizada pelas diretrizes atuais ${ }^{2}$. Além disso, a dose diária deve ser reduzida, ou até feita em dias intercalados, se situações de risco para intoxicação digitálica estiverem presentes, como idade avançada do paciente, deterioração da função renal, massa corporal reduzida, ou uso concomitante de fármacos que interagem com a digoxina (p. ex. claritromicina, eritromicina, amiodarona, itraconazol, ciclosporina, verapamil e quinidina $)^{2}$. Para fins de controle da terapia digitálica, níveis séricos de digoxina deverão ser obtidos rotineiramente nas situações supracitadas ou em casos de suspeita de intoxicação, objetivando-se níveis séricos entre 0,5 e $1,0 \mathrm{ng} / \mathrm{mL}^{2}$.

Sinais de intoxicação digitálica incluem sintomas gastrointestinais (náuseas, vômitos, hiporexia), queixas neurológicas (confusão mental, distúrbios visuais) e 
arritmias cardíacas (extrassistolias, ritmos de reentrada, bloqueios de condução) ${ }^{2}$. Pacientes com sintomas de intoxicação e sinais de arritmia cardíaca, com potencial risco de deterioração clínica, deverão ser conduzidos para a sala de urgência ou Centro de Terapia Intensiva, monitorizados e, se necessário, receber suporte avançado de vida. Se houver algum distúrbio eletrolítico, como hipocalemia e/ou hipomagnesemia, este deverá ser revertido o mais rapidamente possível, em virtude do risco de grave arritmia cardíaca ${ }^{2}$.

\subsubsection{Opções para Terapêutica de Substituição}

Pacientes com IC e intolerantes ao IECA, ou com alguma contraindicação ao uso de IECA ou BRA, beneficiam-se das propriedades vasodilatadoras venosa e arteriolar da combinação de dinitrato de isossorbida e hidralazina. Em baixas doses (até 30 $\mathrm{mg} / \mathrm{dia}$ ), o dinitrato de isossorbida tem efeito preponderantemente venodilatador, reduzindo a pré-carga cardíaca e os sintomas da IC ${ }^{1,2}$. Por outro lado, a hidralazina é vasodilatadora arteriolar exclusiva, tendo ação sobre a pós-carga ao coração. Resultados inconclusivos dos estudos multicêntricos disponíveis geram ainda dúvidas quanto aos benefícios desta combinação de fármacos sobre a mortalidade na IC, visto que ambos são considerados apenas como vasodilatadores e, sem o efeito sobre o eixo neuro-hormonal, não teriam qualquer ação sobre o remodelamento cardíaco ${ }^{1,2,7}$.

As diretrizes nacional e internacional para o manejo de IC recomendam o uso da combinação de dinitrato de isossorbida e hidralazina para todos os pacientes com intolerância ao IECA ou contraindicação ao IECA e/ou BRA (classe de recomendação I) ${ }^{1,2}$. Além disso, benefício adicional desta associação pode ser visto em pacientes afro-americanos, principalmente se eles se mantém sintomáticos apesar do uso otimizado de IECA/BRA e beta-bloqueador (classe de recomendação IIa) ${ }^{1,2}$. De qualquer modo, ao se optar pela introdução de isossorbida (10 mg, 3 vezes ao dia) e hidralazina (12,5 mg, 3-4 vezes ao dia), devese iniciar com doses baixas e fazer o ajuste nas respectivas doses conforme a resposta do paciente.

O dinitrato de isossorbida deverá ser administrado em doses assimétricas, com pausas de mais de 10 horas durante a noite, a fim de se evitar o mecanismo de taquifilaxia. É digno de nota que a hidralazina reduz as chances de taquifilaxia quando em uso concomitante com o nitrato ${ }^{2}$. Além disso, a hidralazina tem efeito vasodilatador adicional durante a fase de compensação de quadro congestivo pulmonar e/ou sistêmico, podendo ser utilizada em associação ao IECA, propiciando melhor perfusão renal neste contexto clínico. Os principais efeitos colaterais dos nitratos são cefaléia e hipotensão arterial, e da hidralazina são hipotensão e taquicardia, este último podendo ser bastante deletério ao paciente, principalmente se ele não estiver em uso de beta-bloqueador ${ }^{2}$.

\subsubsection{Bloqueadores de Canal de Cálcio}

Drogas bloqueadoras dos canais de cálcio podem deprimir significativamente a força de contração do miocárdio, principalmente quando em associação com beta-bloqueador, de modo que são formalmente contra-indicados no manejo da IC sistólica (classe de recomendação III) ${ }^{1,2}$. Além disso, estes agentes podem elevar o risco de arritmias cardíacas deletérias ao paciente ${ }^{2}$. Entretanto, os bloqueadores de canais de cálcio considerados como "vasosseletivos" (p. ex. amlodipina, fenlodipina) não possuem efeito inotrópico negativo, de modo que podem ser utilizados eventualmente como coadjuvantes no controle pressórico de pacientes com IC, sem afetar o prognóstico da doença $^{2}$. Neste caso, a amlodipina é a medicação mais utilizada no Brasil, sendo prescrita em doses únicas diárias de 5 a $10 \mathrm{mg}$ (Tabela 3).

\subsubsection{Medicamentos a serem evitados em pa- cientes com IC}

Alguns medicamentos podem piorar os sintomas da IC, além de exercer efeitos deletérios no tecido cardíaco e renal, e devem ser evitados ou suspensos, caso os pacientes estiverem em uso regular. Estas classes de fármacos são as seguintes: 1) anti-inflamatórios não-esteroidais, que podem causar retenção de sódio e água, vasoconstricção periférica, desequilíbrio de fatores vasoconstrictores coronarianos, e comprometimento da função renal; 2) agentes anti-arrítmicos, que podem exercer significativo efeito pró-arrítmico e depressor do miocárdio (exceção feita à amiodarona); e 3) bloqueadores dos canais de cálcio (vide acima).

\section{Descompensações da IC na en- fermaria}

Os quadros de descompensação de IC geralmente se devem a fatores que causam, de alguma maneira, aumento na pré-carga, diminuição da contratilidade ou aumento na pós-carga cardíaca ${ }^{17}$. Estes eventos podem levar tanto ao aumento na pressão 
capilar pulmonar como à redução no débito cardíaco, ou a ambos, determinando os denominados "perfis hemodinâmicos" do paciente ${ }^{17}$. Independentemente da causa da descompensação da IC, o perfil clínico/ hemodinâmico do paciente permitirá ao médico selecionar a terapêutica (vasodilatadora e/ou diurética) mais adequada à situação encontrada, após o atendimento de suporte já ter sido iniciado, conforme os princípios preconizados no Suporte Avançado de Vida em Cardiologia (SAVC).

Eventualmente, pacientes com IC admitidos na enfermaria do HERP poderão apresentar, em algum momento, quadro de descompensação dos sintomas congestivos, cursando ou não com baixo débito cardíaco. Caberá ao médico plantonista o atendimento de emergência inicial, a conduta terapêutica apropriada e a investigação diagnóstica, devendo o paciente ser encaminhado imediatamente ao Serviço de Emergência em casos de sintomas de baixo débito cardíaco associados aos de congestão (perfil hemodinâmico C - vide referência 17).

A identificação e o manejo dos diferentes perfis clínicos de descompensação cardíaca estão detalhados em outros textos de referência ${ }^{17,18}$, e não se constituem no objetivo do presente estudo.

\section{Protocolo de tratamento propos- to e conclusão}

A figura 1 apresenta o protocolo de admissão e manejo para todo paciente com diagnóstico de IC internado na enfermaria do HERP. Conforme discutido acima, o objetivo da equipe multidisciplinar é o de orientar o paciente quanto à aderência aos tratamentos não-farmacológico e farmacológico. Quanto ao tratamento medicamentoso, a equipe médica deverá reavaliar o esquema terapêutico em uso, suspendendo as medicações deletérias e realizando ajustes nas doses de fármacos que têm impacto tanto na compensação de quadros agudos, quanto na mortalidade a longo prazo. Além disso, a aderência às medicações pode ser abordada pela equipe de Farmacêuticos do HERP, visto que grande parcela dos pacientes atendidos pelo Sistema Único de Saúde é proveniente de condições sócio-culturais desfavoráveis e, geralmente, têm dificuldades na compreensão das receitas médicas. Por fim, ao se aproximar o momento de Alta Hospitalar, os pacientes com IC deverão ser referenciados para os devidos serviços de Cardiologia, garantindo a continuidade do trabalho desenvolvido no HERP.

\begin{abstract}
The increasing prevalence in heart failure and associated nonadherence of patients to the proposed ambulatory-treatment regimen cause the decompensate clinical picture to be the cornerstone of the high incidence of hospitalization found in such disorder, which brings a greater impact in public health. The purpose of the present study is to establish a protocol to be applied to heart failure inpatients at an internal medicine ward, such as the Hospital Estadual de Ribeirão Preto (SP, Brazil), in order to guarantee complete recovery from acute decompensated heart failure, as well as to reassure that patients understand the importance of following the proposed treatment protocols. Accordingly, pharmacological therapy and nonpharmacological strategies will be either reintroduced or optimized, in order to reduce the period of hospitalization, rate of infirmary readmission, and also to favor a better ambulatory follow-up of these patients.
\end{abstract}

Key words: Heart Failure. Patients' Rooms. Clinical Management. 

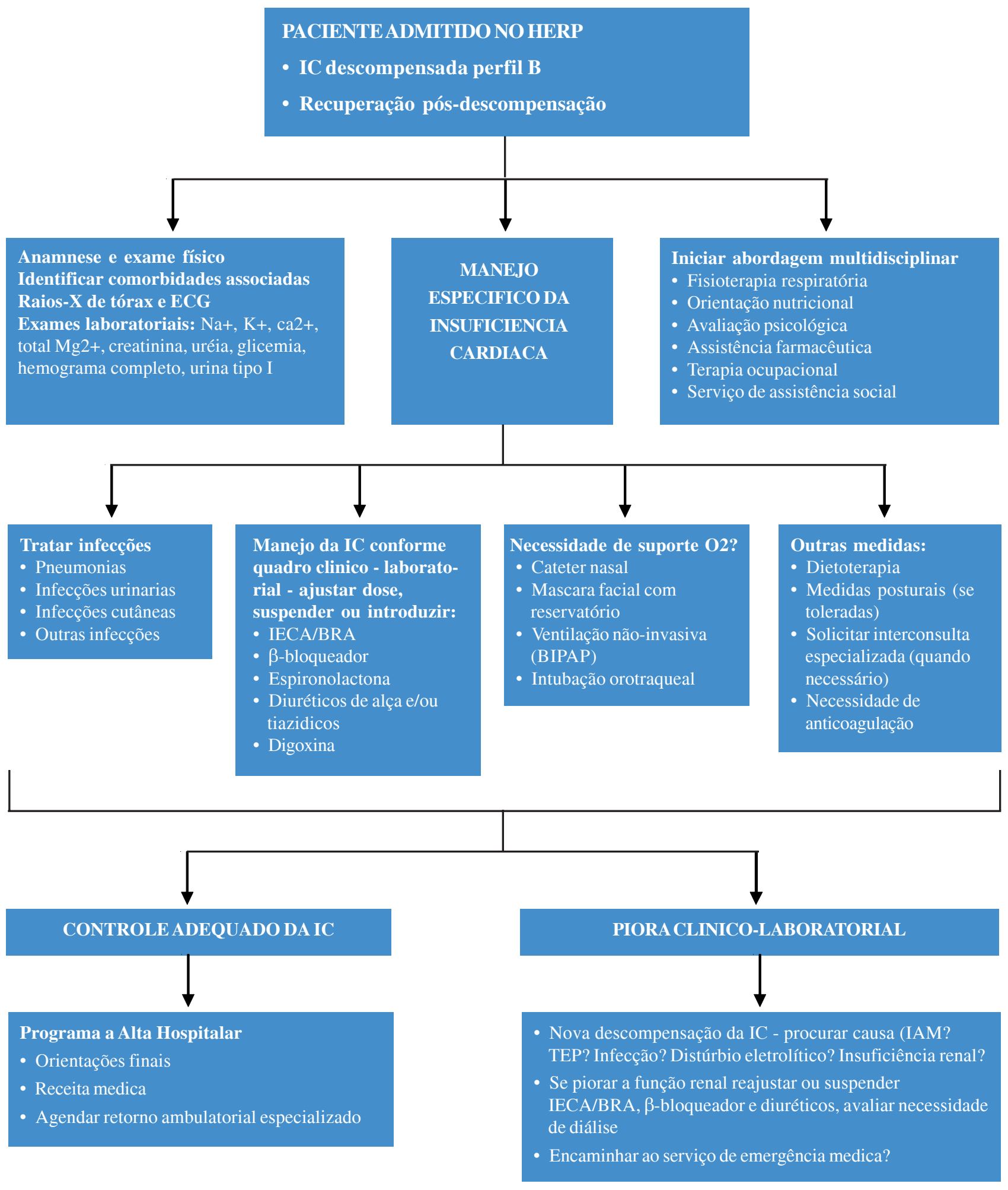

Figura 1: Manejo do paciente internado com Insuficiência Cardíaca. 


\section{Referências Bibliográficas}

1. Bocchi EA, Marcondes-Braga FG, Ayub-Ferreira SM, Rohde LE, Oliveira WA, Almeida DR, e cols. Sociedade Brasileira de Cardiologia. III Diretriz Brasileira de Insuficiência Cardíaca Crônica. Arq Bras Cardiol 2009;92(6 supl.1):1-71

2. Hunt SA, Abraham WT, Chin MH, Feldman AM, Francis GS, Ganiats TG, et al. 2009 Focused Update Incorporated Into the ACC/AHA 2005 Guidelines for the Diagnosis and Management of Heart Failure in Adults. A Report of the American College of Cardiology Foundation/American Heart Association Task Force on Practice Guidelines developed in collaboration with the International Society for Heart and Lung Transplantation. J Am Coll Cardiol 2009; 53(15): e1-e90.

3. Echenique LS, da Rosa Filho R, Lopes RD, Guimarães HP Insuficiência Cardíaca. In: Schor N, Lopes AC, editores. Guias de Medicina Ambulatorial e Hospitalar UNIFESP - Clínica Médica. 1를 Ed; Manole LTDA.; 2007. p. 181-205.

4. Dickstein K, Cohen-Solal A, Filippatos G, McMurray JJV, Ponikowski P, Poole-Wilson PA, et al. ESC guidelines for the diagnosis and treatment of acute and chronic heart failure 2008. Eur J Heart Fail 2008; 10: 933-89.

5. Maeder MT, Kaye DM. Heart failure with normal left ventricular ejection fraction. J Am Coll Cardiol 2009; 51: 905-18.

6. Hess OM, Carroll JD. Clinical Assessment of Heart Failure. In Braunwald's Heart Disease: a Textbook of Cardiovascular Medicine, $8^{\text {th }}$ edition; 2007. p. 561-9.

7. Mann DL. Management of heart failure patients with reduced ejection fraction. In Braunwald's Heart Disease: a Textbook of Cardiovascular Medicine, $8^{\text {th }}$ edition; 2007. p. 611-40.

8. Heart Failure Society of America. HFSA 2006 comprehensive heart failure practice guideline. Section 3: Prevention of ventricular remodeling, cardiac dysfunction, and heart failure. $J$ Card Fail 2006; 12(1): e12-e15.
9. Belardinelli R, Georgiou D, Cianci G, Purcaro A. Randomized, controlled trial of long-term moderate exercise training in chronic heart failure. Effects on functional capacity, quality of life, and clinical outcome. Circulation 1999; 99(9): 1173-82.

10. Hambrecht R, Gielen S, Linke A, Fiehn E, Yu J, Walther C, et al. Effects of exercise training on left ventricular function and peripheral resistance in patients with chronic heart failure: a randomized trial. JAMA 2000; 283(23): 3095-101.

11.Jessup M, Brozena S. Heart Failure. NEJM 2003; 348(20): 2007-18.

12. Krum H, Abraham WT. Heart Failure. Lancet 2009; 373: 94155.

13. Frishman WH. Carvedilol. NEJM 1998; 339(24): 1759-65.

14. Münzel T, Gori T. Nebivolol: the somewhat-different b-adrenergic receptor blocker. J Am Coll Cardiol 2009; 54(16):1491-9.

15.Cohen-Solal A, Kotecha D, van Veldhuisen DJ, Babalis D, Böhm $M$, Coats AJ, et al. Efficacy and safety of nebivolol in elderly heart failure patients with impaired renal function: insights from the SENIORS trial. Eur J Heart Fail 2009; 11: 872-80.

16. Weber KT. Aldosterone in Congestive Heart Failure. NEJM 2001; 345(23): 1689-97.

17. Miranda $\mathrm{CH}$, Castro RBP, Pazin Filho A. Abordagem da descompensação aguda da insuficiência cardíaca crônica. Medicina (Ribeirão Preto). 2003; 36: 179-86.

18. Olmos RD, Correia GF. Insuficiência Cardíaca Congestiva e Choque Cardiogênico no Pronto-Socorro. In: Martins HS, Neto AS, Velasco IT, editores. Emergências Clínicas Baseadas em Evidências. Editora Atheneu; 2006. p. 217-32. 